\title{
Limits of Optical Fibre Communication Systems
}

\author{
Andrew Ellis, Filipe Ferreira, Tingting Zhang, Mohammad Al Khateeb \\ Aston institute of Photonics Technology, Aston University, Aston Triangle, Birmingham, UK \\ Tel: +44 1212043075,e-mail: andrew.ellis@aston.ac.uk
}

\begin{abstract}
In this presentation, we will review the historical evolution of performance predictions for optical communication systems, including single channel systems, soliton systems and high spectral density coherent systems. We will describe how such predictions have been made from the outset of optical communications research and their present form, accurately predicting the performance of coherently detected communication systems and establishing the fundamental limits of nonlinearity compensation.
\end{abstract}

Keywords: nonlinear analysis, optical communications, coherent, four wave mixing.

\section{INTRODUCTION}

Theoretical studies of the performance of optical transmission systems have always sought to establish a practical limit. Since 2009, this limit has been commonly called the "nonlinear Shannon limit" [1-2] and a consensus has begun to form regarding the actual maximum achievable performance. When taking into account the upper limit of the throughput of optical transmission systems and the constant growth (40\% per annum [3]) in traffic demand, an optical "capacity crunch" has now been predicted [4-5]. This will necessitate a shift in the business model for telecommunications service providers. The theoretical prediction of the performance of optical communication systems dates back to the 1960's [6-7]. In this presentation, we will trace their evolution and review their contemporary relevance for both directly and coherently detected systems. We will examine the different models, and extend the analysis to systems which compensate for nonlinear impairments.

\section{NONLINEARITY MODELS IN SINGLE MODE FIBRE}

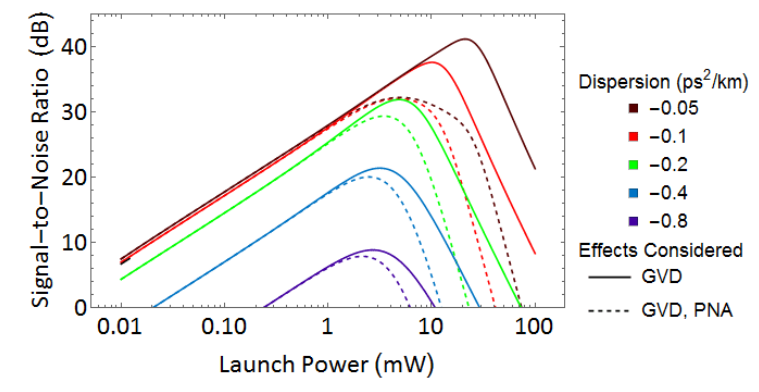

Figure 1. Predicted signal to noise ratio for a 40 Gbaud ASK-DD over ten $50 \mathrm{~km}$ spans.

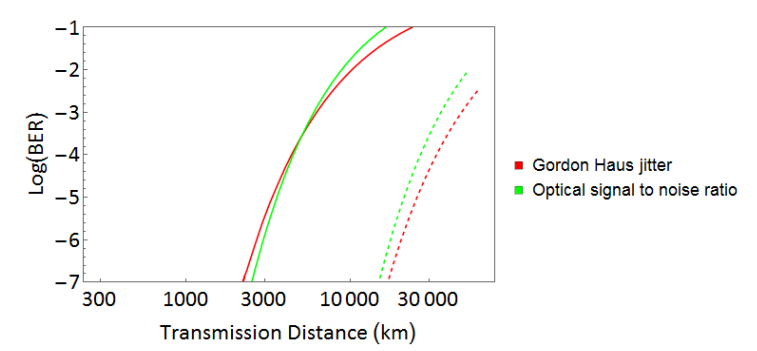

Figure 3. $33 \mathrm{Gbit} / \mathrm{s}$ on-off keyed soliton system, $34 \mathrm{~km}$ spacing and optimised pulse width. Solid: dispersion $16 \mathrm{fs} / \mathrm{nm} / \mathrm{km}$. Dashed: dispersion of $-1 \mathrm{ps} / \mathrm{nm} / \mathrm{km}$ for $32 \mathrm{~km}$ and $16 \mathrm{ps} / \mathrm{hm} / \mathrm{km}$ for $2 \mathrm{~km}$.

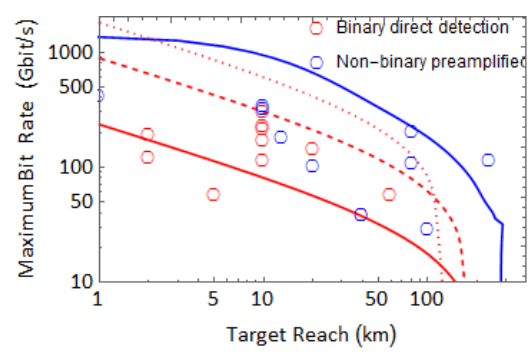

Figure 2. Maximum bit rate for single wavelength systems with direct detection as a function of transmission length over single mode fibre.

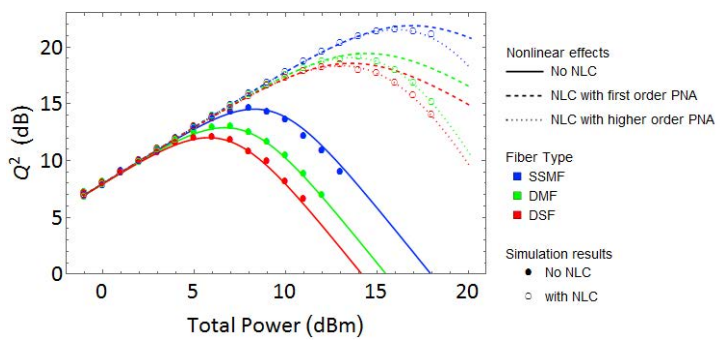

Figure 4. Simulated and predicted performance of lumped system with amplifier noise figure of $6 d B$ and passing 8 channel, 28 Gbaud PM-QPSK Nyquist WDM system over 12 spans of $100 \mathrm{~km}[8]$

Figures 1-4 (adapted from [8]) illustrate the performance predictions of various models including; the combined effects of self-phase modulation and dispersion for single wavelength systems (solid lines, Fig. 1) [9-11], parametric noise amplification (dashed lines, Fig. 1) [12-13], comparison of the predictions from these models with reported direct detection results (Fig. 2, see [8] for references to experimental results), soliton transmission systems with and without dispersion management (Fig. 3) [14-17], and the contemporary integration of four-wave mixing efficiencies under the assumption of Gaussian noise like input signals (Fig. 4) [18-21] with and without nonlinearity compensation (ideal DBP [22]). All of the models presented appear to have remained accurate, provided that the approximations used to derive them are adhered to. For example, 
Fig. 2 compares the predictions from $20^{\text {th }}$ century models alongside $21^{\text {st }}$ century experimental results aimed at data centre applications with no indication that the model is inaccurate.

Careful inspection of these models [8] reveals a consistent theme. For simple systems operating with high dispersion and making no attempt to compensate for the nonlinear effects, it is sufficient to calculate the combined effects of dispersion and nonlinear interactions among the signals. However, for low dispersion systems (Fig. 1-3) or systems with nonlinearity compensation (Fig. 3-4), the nonlinear interaction between the signal and noise must be taken into account in order to attain accurate performance limits. For a system employing in-line optical amplification, differences in growth rates between the various nonlinear interactions impact their relative strength. This is illustrated in Fig. 5 which shows that for single mode fibre systems that deploy 20 to 40 amplifiers and for shorter systems with nonlinearity compensation, parametrically amplified noise [12-13, 21-24] is dominant, but for transoceanic systems, the process of nonlinear phase noise [25] is of increasing significance.

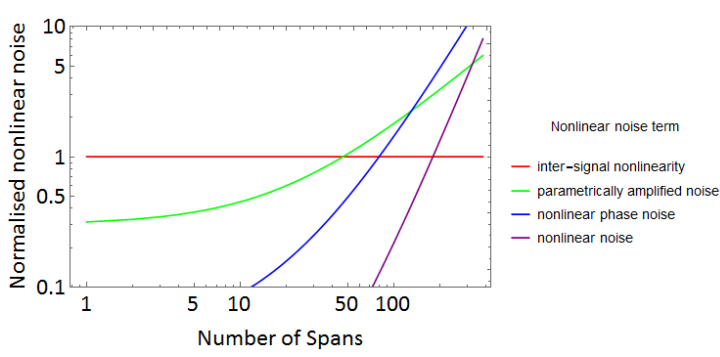

Figure 5. Relative impact of nonlinear interactions involving amplified spontaneous emission, with respect to inter-signal nonlinearity for a $20 \mathrm{~dB}$ optical signal to noise ratio after one amplifier.

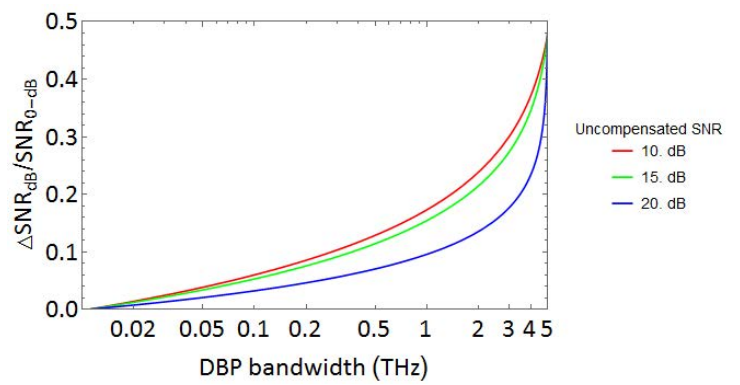

Figure 7. Predicted performance gain (50 spans) for $5 \mathrm{THz}$ systems normalised to the original signal to noise ratio as a function of DSP bandwidth.

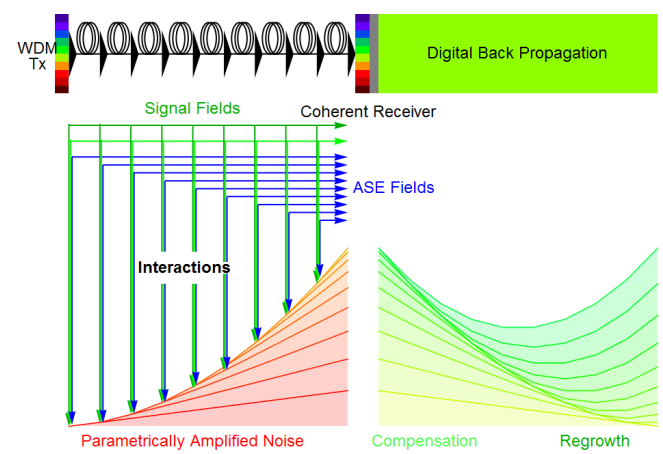

Figure 6. Evolution of parametrically amplified noise in a 9 span system with $D B P$.

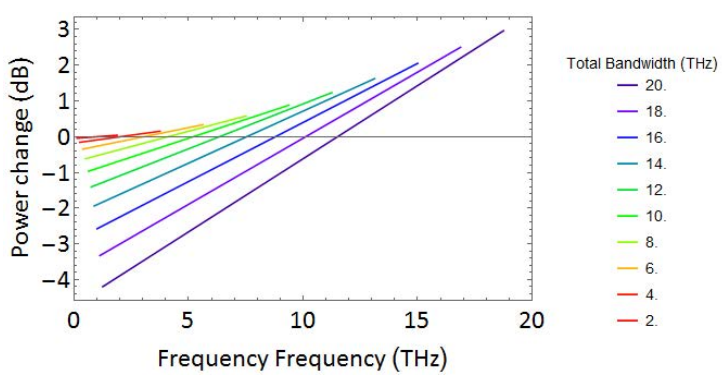

Figure 8. Approximate impact of inter-signal Raman power transfer over a $100 \mathrm{~km}$ Raman amplified span as a function of channel position.

As shown in Fig. 5, the majority of the nonlinear noise in many optical transmission systems is deterministic interaction between signals (assuming point-to-point transmission and cooperation between receivers). Compensating for inter-signal nonlinearity could, in principle, reduce the nonlinear noise by an order of magnitude and so enable higher launch powers and improved system performance [22, 26-27]. For a multichannel system, it is unlikely that a single receiver would process the system and the practical limit in the ability to improve the signal to noise ratio appears to be around $1-2 \mathrm{~dB}$ [28]. However, by resorting to optical combs, significant gains are possible for isolated super-channels propagating without neighbours [29-31], and in this case the system becomes limited by parametric noise amplification. The evolution in this type of nonlinear noise for a system employing digital back-propagation is shown in Fig. 6, which shows clearly that it is not possible to fully eliminate parametric noise amplification. Parametric noise amplification thus sets a (higher) limit on the performance of an optical communication system, where the maximum signal to noise ratio in $\mathrm{dB}$ after ideal nonlinearity compensation is 1.5 times the maximum signal to noise ratio without compensation (less $0.5 \mathrm{~dB}$ to account for higher order nonlinearity) [32]. However, the Shannon limit indicates that increasing the used bandwidth is exponentially more favourable than increasing the signal-to-noise ratio and so systems designers will occupy all available bandwidth before compensating nonlinearity. In the context of a fully populated system, the reach doubling and tripling achieved with isolated super channels is unlikely to be achieved in a fully loaded transmission system unless the superchannel occupies the entire system bandwidth, as shown in Fig. 7.

\section{SCATTERING EFFECTS}

The theoretical models that describe the performance of optical transmission system often concentrate on the modelling of the Kerr nonlinear effects. However, the inelastic nonlinear effects of the optical fiber, such as 
Brillouin and Raman scattering [33], may also degrade the performance of the transmission system. Brillouin scattering limits the signal power spectral density (typically around $0.5 \mathrm{~W} / \mathrm{GHz}$ compared to less than $1 \mathrm{~mW} / \mathrm{GHz}$ in Fig. 4), whilst Raman scattering predominantly results in gain tilts especially in broadband optical transmission systems (Fig. 8), but may also induce nonlinear noise.

\section{NONLINEARITY MODELS IN FEW-MODE FIBRE}

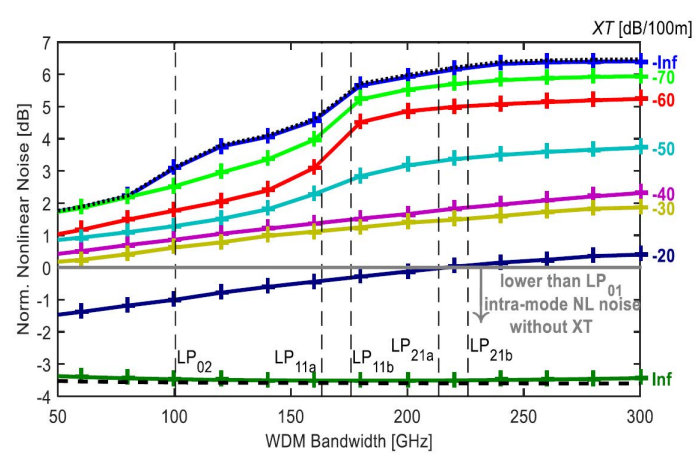

Figure. 9. Total nonlinear noise power in LP01 as a function of WDM bandwidth showing Manakov predictions for strong (dashed) and weak (dotted) crosstalk and with fully stochastic simulations (solid).

To determine the impact of nonlinear interactions in fewmode fibres (FMFs), additional impairments must be considered, namely: differential mode delay, linear mode coupling, and inter-mode nonlinear effects [34]. Generalised Manakov equations may be derived assuming extreme levels of crosstalk, the strong regime being the most beneficial with the SNR per mode scaling with the number of modes $(N)$ as $N^{1 / 3}$ [35]. However, fibres typically operate mainly in the intermediate regime [36] and fully stochastic models which include phase effects have to be considered. This can be achieved using a semi-analytical model capable of solving the coupled differential equations describing the linear mode coupling [37-38]. Studies in the intermediate coupling regime (Fig. 9) show that for coupling strengths beyond $-20 \mathrm{~dB} / 100 \mathrm{~m}$, suppression of nonlinear distortion below that of the isolated mode without mode coupling can be achieved.

\section{PERFORMANCE METRICS}

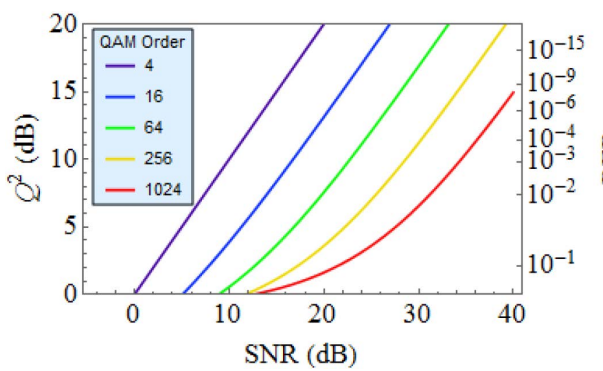

Figure 10. Relationship between performance metrics used in optical fibre communications.
Along with evolution of models analytically predicting the performance of communications systems performance, metrics themselves have evolved. For a digital communication link, the primary figure of merit is the bit error ratio (BER) and the earliest measurements focused on this metric. However, before the prevalence of FEC, systems tests to low BER were required to assure performance, but were particularly time consuming, with 100 errors at $10 \mathrm{Gbit} / \mathrm{s}$ requiring a measurement interval of nearly 3 hours at a BER of $10^{-9}$. $Q$ factor measurements, based on estimations of the tail probabilities of signal distributions, were introduced along with eye mask tests in order to compare products and assure performance. Despite the lack of clear definition for non-binary formats the use of $Q^{2}$ prevails today. Assuming a definition of $Q$ as "that $Q$ factor for a QPSK signal which would give the same BER as the measured system”, Fig. 10 shows the relationship between $Q^{2}$, signal to noise ratio and BER.

\section{CONCLUSIONS}

We have discussed the historical evolution of performance limit predictions. Whilst substantial gains are possible in practice, it has always been the case that the fundamental limit arises from the nonlinear interaction between the signal and noise fields, either in terms of parametric noise amplification, Gordon Haus jitter, or nonlinear phase noise. Practical limits strongly depend on the degree to which the nonlinearity is compensated. For the foreseeable future (until fully populated WDM systems enjoy widespread deployment), it is unlikely that systems will be installed operating anywhere close to the new performance limits, although performance gains of up to $3-4 \mathrm{~dB}$ should be possible.

\section{ACKNOWLEDGEMENTS}

This work is partially funded by; the Engineering and Physical Sciences Research Council (EPSRC) EP/L000091/1-PEACE, EP/M005283/1-UPON); the Royal Society Wolfson Research Merit Award Scheme (WM120035), and by the Marie Skłodowska-Curie Actions (654809-HSPACE). The authors would like to thank M. Tan, M. McCarthy, W. Forysiak, M. Sorokina, F. Ferreira, N. Doran and S. Sygletos for useful discussions.

\section{REFERENCES}

[1] J-C. Antona: Key technologies for present and future optical networks, presented at Topical Workshop on Electronics for Particle Physics 2009 (URL: https://indico.cern.ch/event/49682/contribution/154).

[2] A. D. Ellis et al.: Approaching the non-linear Shannon limit, J. Lightwave Technol., vol. 28, pp. 423-433, 2010. 
[3] A.D. Ellis et al.: Communication networks beyond the capacity crunch, Phil. Trans. R. Soc. A, vol. 374, pp. 20150191.

[4] D.J. Richardson: Filling the light pipe, Science, vol. 330(6002), pp. 327-328, 2010.

[5] A. Chraplyvy: The coming capacity crunch, in Proc. of ECOC 2009, Vienna, Austria, Second Plenary Presentation. (http://ieeexplore.ieee.org/stamp/stamp.jsp?tp=\&arnumber=5287305\&isnumber $=5286960$ )

[6] D. D. Matulka: Application of LASERS to digital communications, IRE Transactions on Aerospace and Navigational Electronics, vol. ANE-9(2), pp. 104-109, 1962.

[7] K. C. Kao and G. A. Hockham: Dielectric-fibre surface waveguides for optical frequencies, Proc. of the Institution of Electrical Engineers, vol. 113(7), pp. 1151-1158, 1966.

[8] A. D. Ellis et al.: Performance limits in optical communications due to fiber nonlinearity, Adv. Opt. Photon., vol. 9, pp. 429-503, 2017.

[9] A. Naka and S. Saito: In-line amplifier transmission distance determined by self-phase modulation and group-velocity dispersion, J. of Lightwave Technol., vol. 12, no. 2, pp. 280-287, 1994.

[10] G. P. Agrawal: Nonlinear Fiber Optics, Second Edition, Academic Press, 1995.

[11] R.S. Vodhanel et al:: Performance of directly modulated DFB lasers in 10-Gb/s ASK, FSK, and DPSK lightwave systems, J. Lightwave Technol., vol. 8, no. 9, pp. 1379-1386, 1990.

[12] C. Lorattanasane and K. Kikuchi: Parametric instability of optical amplifier noise in long-distance optical transmission systems, IEEE J. of Quantum Electronics, vol. 33, no. 7, pp. 1068-1074, 1997.

[13] R. Hui et al.: Modulation instability and its impact in multispan optical amplified IMDD systems: Theory and experiments, J. of Lightwave Technol., vol. 15, no. 7, pp. 1071-1082, 1997.

[14] A. D. Ellis: All optical networking beyond $10 \mathrm{Gbits} / \mathrm{s}$ : OTDM networks based on electro-optic modulators and fibre ring lasers, PhD thesis, Aston University, 1997.

[15] J. P. Gordon and H. A. Haus: Random walk of coherently amplified solitons in optical fiber transmission, Opt. Lett., vol. 11, pp. 665-667, 1986.

[16] D. Wood: Constraints on the bit rates in direct detection optical communication systems using linear or soliton pulses, J. of Lightwave Technol., vol. 8, no. 7, pp. 1097-1106, 1990.

[17] E.M. Dianov et al.: Long-range interaction of picosecond solitons through excitation of acoustic waves in optical fibers, Appl. Phys. B, vol. 54, no. 2, pp. 175-180, 1992.

[18] X. Chen and W. Shieh: Closed-form expressions for nonlinear transmission performance of densely spaced coherent optical OFDM systems, Opt. Express, vol. 18, pp. 19039-54, 2010.

[19] P. Poggiolini: The GN model of non-linear propagation in uncompensated coherent optical systems, J. of Lightwave Technol., vol. 30, no. 24, pp. 3857-3879, 2012.

[20] A.D. Ellis et al.: The impact of phase conjugation on the nonlinear-Shannon limit, in Proc. 2015 IEEE Summer Topicals Meeting 2015, Nassau, Bahamas, 2015, pp. 209-210.

[21] M. A. Z. Al-Khateeb et al.: Effect of second order signal-noise interactions in nonlinearity compensated optical transmission systems, Opt. Lett., vol. 41, pp. 1849-1852, 2016.

[22] X. Li et al.: Electronic post-compensation of WDM transmission impairments using coherent detection and digital signal processing, Opt. Express, vol. 2, pp. 881-888, 2008.

[23] D. Rafique and A. D. Ellis: Impact of signal-ASE four-wave mixing on the effectiveness of digital back-propagation in $112 \mathrm{~Gb} / \mathrm{s}$ PM-QPSK systems, Opt. Express, vol. 19, pp. 3449-3454, 2011.

[24] P. Serena: Nonlinear signal-noise interaction in optical links with nonlinear equalization, J. of Lightwave Technol., vol. 34, no. 6, pp. 1476-1483, 2016.

[25] J. P. Gordon and L. F. Mollenauer: Phase noise in photonic communications systems using linear amplifiers, Opt. Lett., vol. 15, pp. 1351-1353, 1990.

[26] L. B. Du et al:: Digital fiber nonlinearity compensation: Toward 1-Tb/s transport, IEEE Signal Processing Magazine, vo. 31, no. 2, pp. 46-56, 2014.

[27] M. G. Taylor: Coherent detection method using DSP for demodulation of signal and subsequent equalization of propagation impairments, IEEE Photonics Technology Letters, vol. 16, no. 2, pp. 674-676, 2004.

[28] R. Dar and P. J. Winzer: On the limits of digital back-propagation in fully loaded WDM systems, IEEE Photonics Technol. Lett., vol. 28, no. 11, pp. 1253-1256, 2016.

[29] E. Temprana et al.: Beating the nonlinear capacity limit, Opt. Photon. News, vol. 27, no. 3, pp. 30-37, 2016.

[30] N. K. Fontaine et al.: Fiber nonlinearity compensation by digital backpropagation of an entire $1.2-\mathrm{Tb} / \mathrm{s}$ superchannel using a full-field spectrally-sliced receiver, in Proc. ECOC 2013, 2013, paper Mo.3.D.5

[31] G. Liga et al:: On the performance of multichannel digital backpropagation in high-capacity long-haul optical transmission, Opt. Express, vol. 22, no. 24, pp. 30053-30062, 2014.

[32] A. D. Ellis et al.: Impact of optical phase conjugation on the nonlinear Shannon limit, JLT, vol. 35, no. 4, p. $792,2017$.

[33] R.G. Smith: Optical power handling capacity of low loss optical fibers as determined by stimulated Raman and Brillouin scattering, Applied Optics, vol. 11(11), pp. 2489-2494, 1972.

[34] S. Mumtaz: Nonlinear propagation in multimode and multicore fibers: Generalization of the Manakov equations, J. of Lightwave Technol., vol. 31, no. 3, pp. 398-406, 2013.

[35] C. Antonelli et al: Modeling of nonlinear propagation in space-division multiplexed fiber-optic transmission, J. of Lightwave Technol., vol. 34, no. 1, pp. 36-54, Jan. 2016.

[36] F. Ferreira et al: Semi-analytical modelling of linear mode coupling in few-mode fibers, J. of Lightwave Technol., vol. 35 , no. 18, pp. 4011-4022, 2017.

[37] F. Ferreira et al:: Advantages of strong mode coupling for suppression of nonlinear distortion in few-mode fibers, in Proc. OFC 2016, paper Tu2E. 3.

[38] F. Ferreira et al.: Nonlinear distortion in mode delay compensated few-mode fibre spans with intermediate coupling, in Proc. ECOC 2016, paper Tu.2.D.2. 